\title{
Asthma medication use during pregnancy, wheeze and estimated exposure to ambient nitrogen dioxide
}

\author{
To the Editor:
}

Asthma affects $8-9 \%$ of pregnant women in the USA [1]. Since asthma exacerbations during pregnancy can lead to poor pulmonary function, hypoxia and other adverse health outcomes for the mother, fetus and neonate [2, 3], maintaining good control of asthma during pregnancy is recommended for the health of both mother and baby [4]. Reviews of the safety of asthma medication use during pregnancy suggest no or minimal effects on fetal growth and perinatal complications [4-6]. In spite of this, many pregnant women choose to reduce or stop their asthma medications [7].

Asthma severity during pregnancy is influenced by physiological changes $[1,6,8]$ and exposure to asthma triggers such as air pollution [9]. Reducing or discontinuing asthma medication during pregnancy may increase asthma severity $[7,10]$.

Pre-pregnancy asthma severity and its relationship with symptoms and medication use during pregnancy have been examined in this population previously [10]. Here we examine effects of changes in asthma medication use during early pregnancy (gestational months 1 and 2) compared to later pregnancy (gestational months 3-9) on the association between exposures to ambient nitrogen dioxide $\left(\mathrm{NO}_{2}\right)$ estimated at a residence and wheeze. Pregnant women with asthma $(n=602)$ were recruited from 56 obstetric practices and 15 clinics associated with six hospitals in Connecticut and western Massachusetts (USA) $[10,11]$. The Yale University Human Investigation Committee (New Haven, CT, USA) approved this study, and all subjects gave informed consent. Women who reported a physician diagnosis of asthma plus reported either asthma symptoms or use of asthma medications (i.e., rescue medication, including short-acting bronchodilators, and maintenance medication, including inhaled or systemic steroids, cromolyn, theophylline, long-acting $\beta_{2}$ agonists and leukotriene inhibitors) in the year before pregnancy were eligible for inclusion in this analysis. Subjects were questioned about symptom and asthma medication use for each gestational month of pregnancy and about other covariates representing additional risk factors related to pregnancy, demographics, current health status and household exposures. Gestational month observations with complete data were included, a total of 4999 observations. For each gestational month, a traffic-based model [12] was used to estimate residential $\mathrm{NO}_{2}$ exposure, a major component of the traffic-related pollutant mixture often used as a surrogate exposure for this important asthma trigger [13]. The outcome of interest was days of wheeze categorised for each 28-day period as none, $1-7,8-14,>14$ but not daily, and daily.

Asthma medications were coded by type (i.e., rescue or maintenance medication, see list above) and a medication step variable was created for each gestational month defined as " 0 " for no asthma medication use, " 1 " for use of rescue medication only, then regardless of rescue medication use, a value of " 2 ," " 3 ," or "4" was assigned for the use of 1,2 , or 3 or more maintenance medications, respectively. A change in medication use variable was created by comparing the maximum medication step in months 1 or 2 to the maximum medication step in months 3-9: " 1 " represented a reduction and " 0 " represented no change or an increase in medication step.

To allow for repeated measures, we used hierarchical ordered logistic regression models with a random term for subject. Generalised linear mixed models (GLIMMIX in SAS [14]) using a compound symmetry covariance structure were used to examine unadjusted associations between wheeze and covariates (controlling for month of pregnancy). Final models examined health effects of exposures to $\mathrm{NO}_{2}$ on wheeze adjusted for change in medication use, other covariates (age, ethnicity, education level, pre-pregnancy body mass index (BMI), allergic status, tobacco smoke exposure during pregnancy, gas stove use, reported mold or mildew, month of pregnancy) and season of observation (defined as cool months (Oct 15-Apr 15) or warm months).

The proportional odds assumption was satisfied for the five-level wheeze variable. The impact of change in medication use and season on the relationship between exposure and wheeze was explored in adjusted models by including, along with a term for the main effect of $\mathrm{NO}_{2}$, an interaction term for each of these 


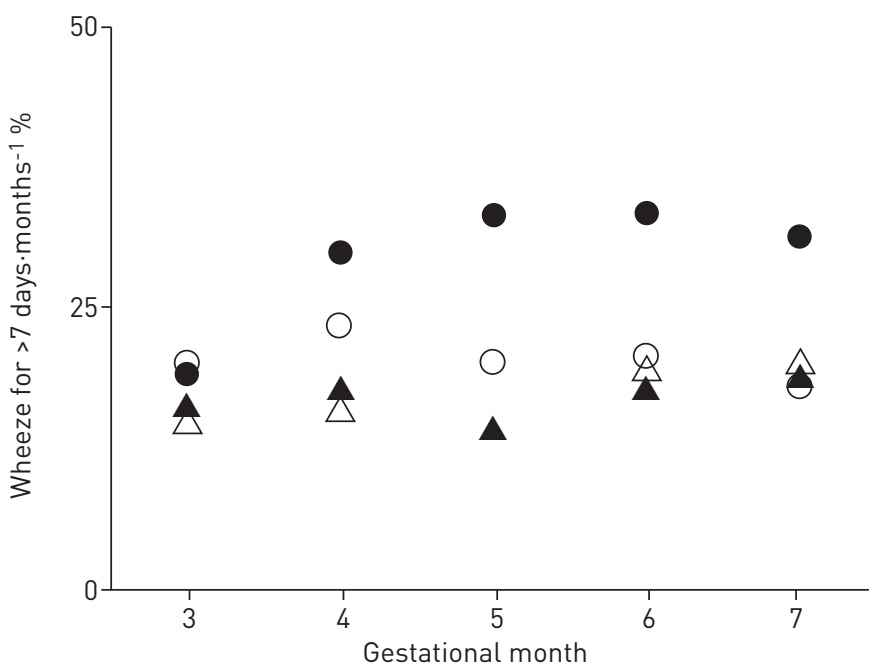

FIGURE 1 Percentage of pregnant women with asthma reporting wheeze for more than 7 days per month for estimated residential exposures to $\mathrm{NO}_{2}$ at levels above (filled symbols) or below (open symbols) 16 ppb during gestational months $3-7$. Women $(n=602)$ were classified according to their use of asthma maintenance medication in months $3-9$ compared to usage in months 1 or 2 : those who reduced their use of medication ( $n=152$; circles) and those who maintained or increased their medication ( $\mathrm{n}=450$; triangles).

variables and $\mathrm{NO}_{2}$. Odds ratios were calculated for the effect of $\mathrm{NO}_{2}$ exposure on the health outcome accounting for different values of interacting covariates.

In models adjusted only for month of pregnancy, risk for increased wheeze was associated with older maternal age: compared with pregnant women younger than 20 years of age, pregnant women aged 35 years or older were 3-times more likely to have increased wheeze (OR 3.37, 95\% CI 1.60-7.11). Compared to white women, Hispanic women were nearly $54 \%$ less likely to report wheeze (OR $0.46,95 \%$ CI 0.29-0.74). Women who reported a history of allergies (OR 2.25, 95\% CI 1.42-3.55) or reported any history of cigarette smoking (OR 2.02, 95\% CI 1.27-3.20) were twice as likely to experience increased wheeze. Increased risk of wheeze was associated with pre-pregnancy obesity (BMI $>29 \mathrm{~kg} \cdot \mathrm{m}^{-2}$; OR 1.72 , 95\% CI 1.08-2.75). Observations from gestational months occurring during the cool months of the year were associated with a $45 \%$ increased risk of wheeze (OR 1.45, 95\% CI 1.26-1.66). Neither years of education, second-hand smoke exposure during pregnancy, gas stove use nor reported mold were significantly associated with days of wheeze. Importantly for the study hypothesis, women who reduced their asthma medication during pregnancy were twice as likely to experience increased days of wheeze (OR 2.17, 95\% CI 1.39-3.39).

The mean \pm SD of the individual $\mathrm{NO}_{2}$ exposure data, used in the health effects analyses from the traffic-based model, was $13.9 \pm 3.4 \mathrm{ppb}$. Figure 1 illustrates the relationship between wheeze, exposure to $\mathrm{NO}_{2}$, month of pregnancy and change in medication use. The percentage of subjects experiencing wheeze in a gestational month is greater for women in the reduced maintenance medication group (fig. 1, circles) compared with women who maintained or increased their level of medication (fig. 1, triangles). This occurred particularly for exposure to $\mathrm{NO}_{2}$ concentrations at or above $16 \mathrm{ppb}$ (mean frequencies $29.5 \%$ versus 17.2\%; fig. 1 filled symbols), less so below $16 \mathrm{ppb}$ (mean frequencies $20.4 \%$ versus $18.5 \%$; fig. 1 , open symbols). The differential effect of $\mathrm{NO}_{2}$ on wheeze across months of pregnancy is seen especially for women exposed to the highest concentration levels $(\geqslant 16 \mathrm{ppb})$ who reduced medication use.

In fully adjusted, ordered logistic regression models, the magnitude of the effect of $\mathrm{NO}_{2}$ was dependent on both medication use and season: parameter estimates were significant for the main effect of $\mathrm{NO}_{2}$ $(\mathrm{p}=0.001)$ as well as for the interaction terms for $\mathrm{NO}_{2}$ and medication use $(\mathrm{p}=0.01)$ and $\mathrm{NO}_{2}$ and season $(\mathrm{p}=0.004)$. For pregnant women who maintained their asthma medication use during pregnancy, a 5-ppb (interquartile interval) increase in $\mathrm{NO}_{2}$ exposure during the warm months resulted in an increased risk for increased days of wheeze (OR 1.68, 95\% CI 1.25-2.26). During this same time period, pregnant women who reduced use of their asthma medication were at more than double the risk of increased wheeze for a 5-ppb increase in $\mathrm{NO}_{2}(\mathrm{OR} 2.57,95 \%$ CI 1.73-3.81). During the cool months, no significant risks for increased wheeze were associated with increases in $\mathrm{NO}_{2}$ exposure for women who maintained their medication use. Women who reduced their medication use were at an increased risk of wheeze during cool months associated with each 5-ppb increase in $\mathrm{NO}_{2}$ concentration (OR 1.81, 95\% CI 1.23-2.67). 
For exposure to spatially heterogeneous, traffic-related pollutants, such as $\mathrm{NO}_{2}$, estimates of locally encountered pollution near roadways is important for understanding associations with health effects [15]. One strength of our study is that our spatially integrated traffic-based model [12] estimated $\mathrm{NO}_{2}$ exposures at a residential level.

In a recent US study of medication dispensed during pregnancy among women with asthma, 63\% filled prescriptions for asthma medication [2]. Over the course of the 6-year study, dispensing maintenance-only medication increased while rescue-only medication dispensing decreased [2], perhaps reflecting the medical community's commitment to treating asthma during pregnancy. One limitation of their study is the inability to measure compliance in medication usage. Although we had no prescription data to assess compliance, in our study, $25 \%$ of the women decreased their asthma medication use.

Most current evidence suggests that benefits of maintaining adequate therapy outweigh risks of adverse outcomes [4-6] and that increased patient education may help pregnant asthmatics overcome their reluctance to maintain treatment $[4,5]$. Reduction of asthma medication use during pregnancy is a potentially modifiable risk that if avoided or reversed could have a beneficial effect on maternal and fetal health.

0 @ERSpublications

Risk of wheeze associated with ambient $\mathrm{NO}_{2}$ exposure is higher for pregnant asthmatics who have reduced their medication http://ow.ly/EBRFJ

Janneane F. Gent, Julie M. Kezik, Melissa E. Hill, Lisa A. McKay, Theodore R. Holford, Brian P. Leaderer and Michael B. Bracken

Center for Perinatal, Pediatric and Environmental Epidemiology, Yale School of Public Health, New Haven, CT, USA.

Correspondence: Janneane F. Gent, PhD, Yale Center for Perinatal, Pediatric and Environmental Epidemiology, One Church St, 6th Floor, New Haven, CT 06510, USA. E-mail: janneane.gent@yale.edu

Received: Sept 022014 | Accepted after revision: Nov 162014 | First published online: Dec 232014

Support statement: This work was supported by grants ES017416, AI41040, DA05484 from the US National Institutes of Health.

Conflict of interest: None declared.

\section{References}

1 Kwon HL, Triche EW, Belanger K, et al. The epidemiology of asthma during pregnancy: prevalence, diagnosis, and symptoms. Immunol Allergy Clin North Am 2006; 26: 29-62.

2 Hansen C, Joski P, Freiman $\mathrm{H}$, et al. Medication exposure in pregnancy risk evaluation program: The prevalence of asthma medication use during pregnancy. Matern Child Health J 2013; 17: 1611-1621.

3 Namazy JA, Murphy VE, Powell H, et al. Effects of asthma severity, exacerbations and oral corticosteroids on perinatal outcomes. Eur Respir J 2013; 41: 1082-1090.

$4 \quad$ McCallister JW. Asthma in pregnancy: management strategies. Curr Opin Pulm Med 2013; 19: 13-17.

5 Gregersen TL, Ulrik CS. Safety of bronchodilators and corticosteroids for asthma during pregnancy: what we know and what we need to do better. J Asthma Allergy 2013; 6: 117-125.

6 Maselli DJ, Adams SG, Peters JI, et al. Management of asthma during pregnancy. Ther Adv Respir Dis 2012: 1-14.

7 Zitstra-van der Woude PA, Vroegop JS, Bos HJ, et al. A population analysis of prescriptions for asthma medications during pregnancy. J Allergy Clin Immunol 2013; 131: 711-717.

8 Hackley B, Feinstein A, Dixon J. Air pollution: impact on maternal and perinatal health. J Midwifery Womens Health 2007; 52: 435-443.

9 Holguin F. Traffic, outdoor air pollution, and asthma. Immunol Allergy Clin North Am 2008; 28: 577-588.

10 Belanger K, Hellenbrand ME, Holford TR, et al. Effect of pregnancy on maternal asthma symptoms and medication use. Obstet Gynecol 2010; 115: 559-567.

11 Bracken MB, Triche EW, Belanger K, et al. Asthma symptoms, severity, and drug therapy: a prospective study of effects on 2205 pregnancies. Obstet Gynecol 2003; 102: 739-752.

12 Skene KJ, Gent JF, McKay LA, et al. Modeling effects of traffic and landscape characteristics on ambient nitrogen dioxide levels in Connecticut. Atmos Environ 2010; 44: 5156-5164.

13 Gauderman WJ, Avol E, Lurmann F, et al. Childhood asthma and exposure to traffic and nitrogen dioxide. Epidemiology 2005; 16: 737-743.

14 SAS Institute Inc. SAS statistical software, version 9.3. SAS Institute, Inc, Cary, NC, 2010.

15 Kunzli N. Effects of near-road and regional air pollution: the challenge of separation. Thorax 2014; 69: 503-504. 